\title{
Prácticas de liderazgo de los directores noveles para la inclusión educativa
}

\author{
María Verónica Leiva Guerrero $\left(^{*}\right)$, Joaquín Gairin Sallán ${ }^{* *}$ y Sebastían Guerra San Martín (*) \\ (*) Pontificia Universidad Católica del Valparaiso - Chile, $\left({ }^{* *}\right)$ Universidad Autónoma de Barcelona - España
}

\section{RESUMEN}

Este artículo se enmarca en una investigación mayor, proyecto Fondecyt que refiere a cómo los directores que están ejerciendo el cargo por primera vez dan respuesta a las necesidades derivadas de la inclusión educativa en sus centros escolares. Para ello, se realizó un estudio de Casos Múltiples, de tipo transversal, con aplicación de una entrevista en profundidad a 9 directores nóveles de centros escolares municipales de la región de Valparaíso. Los resultados indican que las prácticas de liderazgo inclusivo se sitúan en cuatro dimensiones: rediseño de la organización con foco en lo inclusivo, desarrollo profesional inclusivo, atención a los aprendizajes diversos e instalación de una cultura inclusiva. Siendo la dimensión rediseño de organización, con foco en lo inclusivo, la que presentó mayor presencia. Para estos directores nóveles, tiene gran importancia poseer un clima laboral que permita la cohesión de las percepciones de los miembros de la comunidad escolar, respecto de su trabajo y rol social institucional.

Palabras Clave: Liderazgo, inclusión, director, novel, prácticas.

\section{Leadership practices of noveles principals for educational inclusion}

\section{ABSTRACT}

This article is part of a larger investigation, project, and refers to inclusive leadership practices in novel principals, in terms of what they do, why and which purpose they have for implementing these practices. In order to achieve that objective, we carried out a Multiple Case/Collective Case Study of transversal nature by applying an in-depth interview to 9 novel principals of public Elementary and High Schools from the Valparaíso region. Data were processed through the Discourse analysis technique. The most relevant findings noted that inclusive leadership practices are situated in four dimensions: organization redesign with a focus on inclusive issues, inclusive professional development, attention to diverse learning and establishment of an inclusive culture in the center. The dimension of organization redesign with a focus on inclusive issues showed the most presence in terms of assigning functions to the existing work teams, modifying the regulations about school coexistence and creating the conditions for collaborative work. It is very important for novel principals to have a work environment that will allow for the cohesion of the perceptions that members of the school Community have regarding their job and their social role in the institution.

Keywords: Leadership, inclusion, principal, novel, practices.

\section{Introducción}

Las políticas educativas nacional e internacional han enfatizado la relevancia de brindar una educación inclusiva que atienda la diversidad de estudiantes. Hacerlo, implica un liderazgo centrado en las personas, percibir la comunidad educativa como sistema de relaciones interdependientes, transformar la cultura más allá de las puertas del centro escolar, involucrar a toda la comunidad y entender que la atención a la diversidad conlleva cambios desde la gestión del currículum, a las prácticas y el liderazgo del director (Carrasco \& González, 2017; Gómez-Hurtado, 2014).

Chile publica en 2010 la Ley 20.422, que establece normas sobre igualdad de oportunidades e inclusión social de personas con discapacidad, asegurando el disfrute de derechos y eliminando cualquier discriminación originada sobre la discapacidad y otras vulnerabilidades.

El artículo 36 de dicha Ley señala que los establecimientos de enseñanza regular deberán incorporar innovaciones y adecuaciones curriculares e infraestructura y materiales de apoyo necesarios para permitir y facilitar a las personas con discapacidad el acceso, brindándoles los recursos adicionales para asegurar su permanencia y progreso en el sistema educacional.

En el año 2015, al amparo de la Ley 20.422, se publica el decreto $\mathrm{N}^{\circ} 83$, que aprueba criterios y orientaciones de adecuación curricular para estudiantes con necesidades educativas especiales de educación parvularia y educación básica. Este decreto se basa en cuatro principios: 1) igualdad de oportuni- 
dades, 2) calidad educativa con equidad, 3) inclusión educativa y 4) valoración de la diversidad y flexibilidad en la respuesta educativa.

Bajo estos postulados legales se encuentra actualmente la educación chilena en materia de inclusión, que espera avanzar hacia una real educación inclusiva, con justicia social y de calidad para todos los estudiantes.

Hoy en día, se da como probado que, entre los elementos internos de la escuela, y después de la labor del profesor en el aula, el liderazgo es el segundo factor en influencia sobre el aprendizaje después del papel del profesor en el aula (Day et al., 2010; Hallinger \& Wang, 2015; Robinson, Hohepa \& Lloyd, 2009; Robinson, Lloyd \& Rowe, 2008). Más aún, algunos autores hablan de una incidencia del 25\% del director en los aprendizajes de los estudiantes (Bolívar, López \& Murillo, 2013).

Por ello, es necesario contar con directores/as que lideren centros educativos para la inclusión, pues

La escuela del siglo XXI se ve obligada a educar y a enseñar en un contexto cultural condicionado por los valores de la participación, la tolerancia, la igualdad, el respeto a los derechos del individuo y la aceptación de la diversidad personal, entre otros (Fernández \& Hernández, 2013).

Tarea no exenta de dificultades para los líderes escolares, si consideramos que se mueven en contextos complejos (Gairín, 2010), donde deben implementarse políticas educativas como las de inclusión, que implican hacerse cargo de la multiculturalidad, las barreras al aprendizaje en grupos vulnerables, la participación de toda la comunidad educativa, y el trabajo colaborativo. También, si consideramos que la promoción de escuela inclusiva es un reto social y educativo actual, que se vincula al binomio calidad y equidad y que se relaciona con los movimientos que demandan justicia social.

En este contexto, tiene sentido indagar sobre cómo los directores que están ejerciendo el cargo por primera vez, dan respuesta a las necesidades derivadas de la inclusión educativa en centros escolares en el marco de la normativa actual.

\section{La inclusión en el sistema escolar}

La escuela reproduce las desigualdades sociales al reforzar el 'habitus' que corresponde a familias con determinada jerarquía social, el acceso al capital cultural y/o nivel económico existente (Bourdieu, 2005; Simón \& Barrios, 2019). La escuela no es el lugar donde se producen las desigualdades sociales, sino donde se legitiman (Reves, 2002). "La exclusión escolar es el preludio de algunas trayectorias de exclusión social" (Parri$\underline{11 a, 2007, p .178)}$ y, desde esta perspectiva, según Martínez-Figueira (2013), lejos estamos de responder a la idea de inclusión educativa que señalaba la Organización de las Naciones Unidas para la Educación, la Ciencia y la Cultura (UNESCO, 2005) para conseguir la equidad y cohesión social que permita responder a sectores excluidos.

Otro aspecto vinculante es aquel desprendido desde la educación especial en un ámbito más clínico y terapéutico (Informe Warnock, 1978; Rodríguez \& Ossa, 2014), donde se atienden las discapacidades cognitivas y físicas como problemas de la persona. Estas políticas implementadas en favor de la integración escolar, preconcebidas inicialmente como inclusivas, han reducido el concepto de diversidad a aquellos estudiantes con necesidades educativas especiales (NEE). Lo que conlleva una forma de exclusión solapada en un concepto discutible de integración (Apablaza, 2014).
Al momento de analizar un programa teórico que integre la inclusión educativa, es importante plantear la necesidad de abordar la escuela desde una perspectiva más abierta a la diversidad, dejando atrás las implicancias de la integración (Ossa, 2014) y, en consecuencia, sumando al análisis una mayor comprensión de la variabilidad que existe en el aprendizaje de los estudiantes, observando, también, las diferencias de otras variables sociológicas como, etnicidad, lenguaje, género, territorio, orientación sexual, entre otras. En contraste con la perspectiva de la integración, el concepto inclusión educativa es mucho más profundo, "la enseñanza se adapta a los alumnos y no estos a la enseñanza" (Blanco, 2006, p. 6).

La inclusión en el campo educativo ha aportado múltiples definiciones en torno a su conceptualización (Martínez-Figueira, 2013), que explican sus diferencias en términos del área desde la cual se desarrollan; no obstante, pareciese existir consenso en las características principales vinculadas a promover y crear una sociedad inclusiva, basada en los valores de justicia social, equidad y participación democrática (Ainscow, 2006; Arnáiz, 2003, 2010; Echeita 2007, 2010; Laluvein, 2010; Marcelo, Murillo, Sánchez, Mayor \& Rodríguez, 2010).

El Centro de Estudios de Educación Inclusiva del Reino Unido (CSIE, 2006) señala que la educación inclusiva debe lograr reducir la exclusión de las culturas, del currículo y de la comunidad de los colegios locales. La inclusión "está ligada a cualquier tipo de discriminación y exclusión, en el entendido de que hay muchos estudiantes que no tienen igualdad de oportunidades educativas ni reciben una educación adecuada a sus necesidades y características personales" (Booth \& Ainscow, 2012, p. 22).

Una dificultad en el desarrollo del discurso inclusivo también reside en la operacionalización de esta categoría analítica. Es necesario generar una descripción profunda de sus conductas y dimensiones, permitiendo así transponerlas a la realidad social y contextos específicos en que se desarrolla (Echeita \& Ainscow, 2011).

Booth y Ainscow (2012) plantean tres dimensiones fundamentales: A) culturas inclusivas, que refieren a la creación de una comunidad acogedora, segura, que genere una colaboración entre sus actores; B) políticas inclusivas, sobre el aseguramiento de la inclusión como centro de desarrollo del centro, es decir, es el soporte institucional, y; C) prácticas inclusivas, que reflejan la cultura y políticas, siendo las prácticas las encargadas de asegurar actividades y acciones, mediante movilización y ejecución de recursos que promuevan la participación y consideren la diversidad del alumnado.

De todas formas, el discurso y la práctica de la inclusión educativa ha sido superadora del tratamiento de la diversidad, pero, esperamos, que en el futuro sea superado por el principio de normalización social. Soñamos con el futuro de una sociedad inclusiva que atiende y respeta a todas las personas en sus particularidades y no precisa de medidas específicas a nivel educativo porque ya están implantadas a nivel social.

La intervención a nivel educativo y en otras instancias (normas de apoyos a grupos desfavorecidos) actualmente tiene sentido en la medida que hablamos de personas y colectivos vulnerables sobre los que intervenimos, para evitar que entren en procesos de discriminación y marginación que implicarían procesos de exclusión difícilmente reversibles (figura 1). También, por entender que la educación puede ayudar a proporcionar la prevención que disminuya los efectos de malas prácticas sociales. 


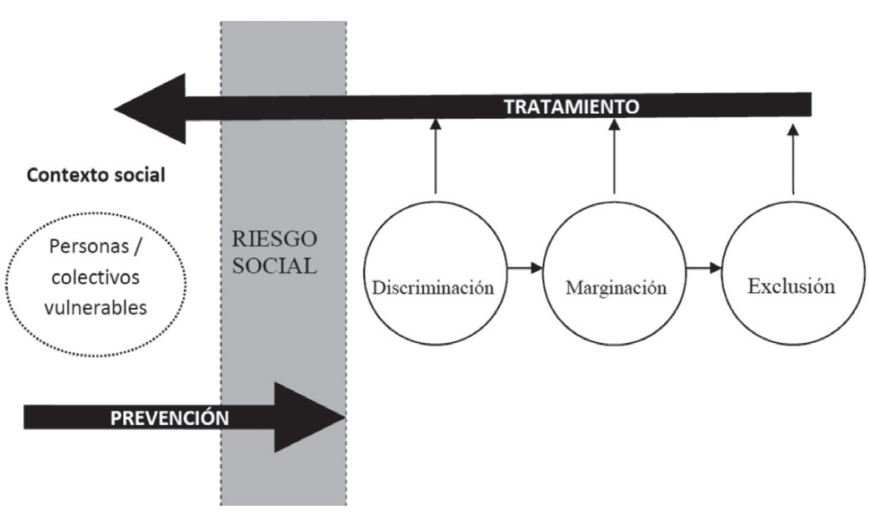

Figura 1. La atención a colectivos vulnerables. Fuente: Gairín, 2015, p.17)

\section{Liderazgo Inclusivo}

El problema de la inclusión educativa genera la necesidad de analizar modelos y enfoques existentes respecto del liderazgo educativo y su relevancia con relación a la especificad de la temática. Por liderazgo educativo entenderemos aquello que dota de un sentido común a la organización escolar e influye en el comportamiento de sus miembros, teniendo como norte la mejora de la calidad, que se expresa tangiblemente en los aprendizajes de los estudiantes (Robinson et al., 2009).

El liderazgo educativo tiene dos funciones principales: establecer una meta común e influir al resto de los actores de la comunidad escolar (Leithwood \& Louis, 2011). Este ejercicio de empoderamiento debe garantizar la instalación de procesos continuos de liderazgo y apropiación en la institución donde se desarrolla. Las competencias y habilidades de liderazgo se transforman en una base para que el director logre implementar prácticas pedagógicas e inclusivas de calidad (Bolívar et al., 2013).

El liderazgo se observa como elemento fundamental para el desarrollo de la escuela en su totalidad, pero ¿qué características debe poseer un liderazgo centrado en la inclusión?

En primera instancia, es necesario señalar las aportaciones que han generado el liderazgo distribuido y colaborativo como complemento al enfoque inclusivo. Este reconoce y asume que existen muchas más personas implicadas y relacionadas en las prácticas de liderazgo. En consecuencia, el liderazgo es compartido y descentralizado, delegando responsabilidades al conjunto de la comunidad, generándose de este modo más liderazgos, que se accionan como una red colaborativa (Spillane, 2005).

El liderazgo distribuido constituye un aporte para este estudio, en la medida que trata de un marco conceptual y analítico para estudiar las interacciones y relaciones subyacentes a los procesos de liderazgo, y además está profundamente interesado en las formas de distribución y patrones característicos de cada espacio de organización (Gronn 2002; Spillane, Halverson \& Diamond, 2004).

\section{Prácticas de liderazgo inclusivo en escuelas}

Las prácticas que definen a un liderazgo inclusivo, no sólo se relacionan con el reconocimiento de enfoques de liderazgo distribuido y colaborativo, sino que también reconocen la capacidad de adquirir y manejar competencias inclusivas. Pero, además, es necesario que los líderes establezcan las condiciones necesarias para promover el logro escolar, la justicia y la equidad como preceptos de la comunidad educativa (Leithwood, 2009; Murillo, Krichesky, Castro \& Hernández, 2010). El liderazgo parece ser aún más importante en escuelas donde atienden estudiantes diversos, y excluidos que, por consiguiente, requieren de prácticas diferenciadas. De todas formas, se ha llegado al consenso de que no existen prácticas específicas que caracterizan a directores inclusivos, tampoco un tipo de liderazgo particular clave para la inclusión. Por el contrario, un líder inclusivo debe estar dispuesto a cambiar estilos y estrategias, para experimentar los que funcionen mejor, considerando sus contextos educativos específicos (Figueroa \& Muñoz, 2014; Muijs, Harris, Chapman, Stoll, \& Russ, 2004).

Por tanto:

Un liderazgo para la inclusión educativa ha de ser un liderazgo democrático y participativo, en el que ocupan un lugar importante las dinámicas de indagación abierta, comunicación, dialogo, colaboración, trabajo en grupo en un marco de respeto en el que necesariamente confluirán perspectivas no siempre consensuales, a veces conflictivas, sobre las que hay que optar desde una base moral (Gonzá$\underline{\text { lez, 2008) }}$

Es importante apreciar que el liderazgo inclusivo debe comprenderse desde una serie de prácticas que pueden ser ejecutadas en forma flexible, desigual y/o combinada. Sin embargo, los valores asociados a la inclusión educativa se apoyan en prácticas directivas, tales como (Gómez-Hurtado 2012; Leithwood \& Riehl, 2005; Murillo \& Hernández, 2011):

A)Visualizarse como promotores de prácticas inclusivas; B) Fomentar el crecimiento de la comunidad educativa, potenciado una cultura de apoyo y colaboración mutua entre los distintos actores; C) Trabajar por una cultura inclusiva, donde exista una serie de valores y actitudes compartidos por el conjunto de los actores de la comunidad educativa; D) Favorecer procesos de enseñanza y aprendizaje inclusivos, que busquen soluciones innovadoras para docentes y se inquieten con el aprendizaje de los estudiantes; E) Colaborar en la relación familia y escuela; y, por último, F) Revalorizar a los estudiantes.

\section{Método}

El estudio se circunscribe al paradigma Descriptivo-Interpretativo (Valles, 1999), dado que sus objetivos buscan conocer una realidad social determinada, salvaguardando las subjetividades y experiencias que los directores noveles han desarrollado mediante aprendizajes situados, siendo el enfoque cualitativo el sustento del estudio. El Diseño es un Estudio de Casos Múltiples (Merrian, 2002) de tipo transversal.

\section{Participantes}

La Población se compone por directores/as de centros escolares pertenecientes a direcciones de administración de educación municipal (DAEM) o corporaciones municipales, que fueron seleccionados mediante el sistema de Alta Dirección Pública (ADP) para ejercer el cargo directivo por primera vez en su carrera desde el primer semestre del año 2014. Los participantes son 9 directores noveles de la región de Valparaíso-Chile que fueron seleccionados por medio de una muestra razonada (Guerrero \& Urbano, 2007). 
Tabla 1.

Caracterización Directores

\begin{tabular}{lllll}
\hline Establecimiento & Género & Título profesional & Grado de Magíster & Experiencia previa \\
\hline Escuela 1 & Femenino & Profesor de Ed. Gral. Básica & Sí & DAEM/UTP/Consultora LEM \\
Liceo 1 & Femenino & Profesora de Matemáticas & Sí & UTP/ Directora interina \\
Escuela 2 & Masculino & Profesor Ed. Física & Sí & Jefe Departamento / Director/Académico \\
Liceo 2 & Femenino & Profesora Ed. Básica y Periodista & No & UTP \\
Liceo 3 & Masculino & Profesor Ed. Técnico Profesional & Sí & Coord. Académico CFT / Encargado Área Técnica FIDE \\
Escuela 3 & Femenino & Profesora Ed. Gral. Básica & Sí & UTP \\
Escuela 4 & Femenino & Profesora Ed. Gral. Básica & Sí & Profesora Encargada \\
Escuela 5 & Femenino & Profesora de Francés/Básica & Sí & UTP/Orientadora \\
Escuela 6 & Femenino & Profesora de Historia y Geografía & Sí & Consultora \\
\hline
\end{tabular}

Fuente: Leiva, Montecinos, Ahumada, Campos \& Guerra, 2016.

\section{Instrumentos y Procedimientos}

Al término del segundo semestre del tercer año en el cargo de Director, cada director participó de una entrevista en profundidad (Alonso, 1998) de duración aproximada de 90 minutos. La entrevista fue grabada en audio y luego transcrita para su análisis.

\section{Análisis de los datos}

Para analizar cómo los directores que están ejerciendo el cargo por primera vez y dan respuesta a las necesidades derivadas de la inclusión educativa en centros escolares, se utilizó el concepto de prácticas para la inclusión educativa declaradas en el Índice de Inclusión (Booth \& Ainscow, 2012), que deben reflejar la cultura y políticas inclusivas implementadas por la escuela, mediante actividades y acciones, tanto fuera como dentro del centro, que promuevan la participación diversificada del alumnado y de la comunidad educativa.

Tabla 2.

Definición de dimensiones de Liderazgo Inclusivo
Se utilizó la técnica de Análisis de Contenido Temático (Mayring, 2000). Los criterios de validez para este artículo son: a) Declaración previa de "habla situada"; b) Construcción teórica y determinación del nivel de saturación de la información proporcionada por los sujetos de la investigación; d) Validación de contenido del protocolo de preguntas en base al juicio experto de tres docentes directivos con amplia experiencia y reconocido currículo profesional a nivel de establecimientos públicos de la comuna de Quilpué, Chile.

\section{Resultados}

Los resultados de esta investigación de acuerdo con lo que declaran hacer los directores nóveles (tabla 2), dan cuenta que las prácticas de liderazgo para la inclusión educativa se identifican en base a cuatro dimensiones:

- rediseño de la organización con foco en lo inclusivo,

- desarrollo profesional inclusivo,

- atención a los aprendizajes diversos e

- instalación de una cultura inclusiva en el centro,

\begin{tabular}{ll}
\hline \multicolumn{1}{c}{ Áreas de Liderazgo } & \multicolumn{1}{c}{ Definición } \\
\hline Rediseño de la organización con foco inclusivo. & $\begin{array}{l}\text { Corresponde a las prácticas relacionadas con reflexionar y ejecutar ajustes } \\
\text { en la organización escolar, en el equipo directivo y en su cuerpo docente, } \\
\text { de acuerdo a las demandas del contexto socio educacional y los estánda- } \\
\text { res institucionales para un desempeño acorde al resguardo de los derechos } \\
\text { educacionales. }\end{array}$ \\
\hline Desarrollo Profesional Inclusivo. & $\begin{array}{l}\text { Se circunscribe a prácticas que buscan relevar conocimientos, actitudes y } \\
\text { recursos al servicio de un modelo inclusivo en todos los funcionarios que } \\
\text { trabajan en el interior de los establecimientos educacionales. Un buen di- } \\
\text { rector debe valorar, potenciar y cualificar el trabajo de los docentes (Weins- } \\
\text { tein, 2009). }\end{array}$ \\
\hline Atención a los aprendizajes diversos. & $\begin{array}{l}\text { Considera prácticas asociadas a trabajar con todo el estudiantado, promo- } \\
\text { viendo el logro escolar, la justicia y la equidad (Leithwood, 2009). }\end{array}$ \\
\hline Instalación de una cultura inclusiva en el centro. & $\begin{array}{l}\text { Se refiere a prácticas que buscan instalar un sello inclusivo a través de la } \\
\text { promoción de la atención de la diversidad, la modificación de los procedi- } \\
\text { mientos relacionados con la convivencia escolar activados en el enfoque de } \\
\text { derechos y en establecer una visión compartida respecto a lo que se debiese } \\
\text { comprender por inclusión educativa, apelando sensibilizar sobre el respeto } \\
\text { de la diversidad (Echeita \& Ainscow, 2011; UNESCO, 2011). }\end{array}$ \\
\hline
\end{tabular}




\section{Rediseño de la organización con foco inclusivo}

Se observan prácticas asociadas al área rediseño de la organización con foco inclusivo (45), destacándose aquellas relacionadas con distribuir funciones entre miembros ya existentes en la institución, que implica no excluir integrantes, salvo casos excepcionales cuando han sido disruptores del ambiente organizacional. Los directores nóveles no hacen mayores cambios, dada la rigidez y las complejidades contractuales del sistema de educación pública. Estas prácticas que buscan dar oportunidades a quienes ya conforman los equipos de gestión, genera críticas por su rigidez, que dificulta la oportunidad por modificar responsabilidades y distribuir roles desde un enfoque propio del liderazgo distribuido (Spillane, 2005).

Los directores apelan a no realizar cambios repentinos en las culturas organizacionales, esperando que se ajusten a parámetros y objetivos que ellos buscan permear en la organización escolar. No obstante, destaca también otra práctica que menciona el ajuste a la organización desde el enfoque de derechos, abocándose principalmente a garantizar normativa y protocolarmente los derechos de estudiantes y funcionarios mediante la gestión de la convivencia, construyendo equipos especializados que garanticen la permanencia o retención escolar y los ambientes propicios para el aprendizaje.

Las prácticas de los directores en torno a influir mediante la adquisición de un enfoque de derechos están directamente asociadas a influir mediante nuevas políticas institucionales en el ejercicio de interacciones entre miembros de la comunidad educativa a través del trabajo colaborativo y la aceptación a las diferencias.

\section{Desarrollo Profesional Inclusivo}

La segunda dimensión que presenta mayor cantidad de referencias (30) es la atención a los aprendizajes diversos que se circunscriben a "prácticas de ejecución directa en estudiantes focalizados", encontrándose referencias sobre estudiantes que están en situación de discapacidad y sobre el apoyo personalizado a estudiantes con dificultades conductuales y/o en situación de discapacidad, a través de acciones sobre infraestructura, espacios físicos adecuados y diversificar el currículum por parte del director y profesores. Además de prácticas dirigidas a la participación de todo el estudiantado en acciones vinculadas a la convivencia escolar, democracia y relaciones dialógicas.

\section{Atención a los aprendizajes diversos}

La tercera dimensión, con 22 referencias, es instalar una cultura inclusiva en el centro. Los directores reconocen que las instituciones a las cuales han llegado no se han caracterizado por tener un sello asociado al ámbito de la inclusión, sino, más bien, recién se encuentran en fase de sensibilizar e instalar prácticas reconocidas desde este enfoque. Estas prácticas desde la mirada de los directores en estudio deben consolidarse desde un proyecto educativo institucional de tipo multifactorial.

\section{Instalación de una cultura inclusiva en el centro}

La cuarta dimensión, con 20 referencias, hace referencia a la formación interna, pero formal de docentes en el Decreto 83/2015 $\mathrm{y}$, en particular, respecto al Diseño Universal del Aprendizaje. De forma crítica se comenta una ausencia en declaraciones de prácticas relacionadas con este ámbito, que tengan vínculo con la evaluación inclusiva y adecuaciones curriculares, que son un componente del espíritu del decreto. Este profundiza en las prácticas de apoyo específico para estudiantes que requieran adecuar sus objetivos de aprendizaje, elevando la complejidad en estudiantes que se encuentren en niveles superiores, así como reduciendo o modificándolos en estudiantes que posean dificultades en el aprendizaje. Los directores señalan, además la necesidad de apoyos para el desarrollo de fortalezas y recursos emocionales y sociales en funcionarios.

Finalmente, se destaca la instalación de espacios de reflexión del quehacer profesional docente, como situaciones de autoformación continua de atención a la diversidad. El construir espacios de comunidades de aprendizaje entre pares, junto con el trabajo colaborativo, son, sin duda, los aspectos más trascendentales de mejoramiento en contextos desfavorables.

Tabla 3.

Dimensiones de las Prácticas de liderazgo inclusivo en discursos de directores noveles.

\begin{tabular}{|c|c|c|c|c|}
\hline Dimensiones & $\begin{array}{c}\text { Prácticas de } \\
\text { Liderazgo } \\
\text { Inclusivo }\end{array}$ & Justificación ¿Por qué? & $\begin{array}{c}\text { Fines/objetivos } \\
\text { ¿Para qué? }\end{array}$ & Ejemplo extracto de transcripción \\
\hline $\begin{array}{l}\text { Rediseño de la } \\
\text { organización con } \\
\text { foco inclusivo } \\
(45)\end{array}$ & $\begin{array}{l}\text { Distribuir } \\
\text { funciones entre } \\
\text { los miembros } \\
\text { ya existentes en } \\
\text { la institución } \\
\text { (4 referencias } \\
\text { directas) }\end{array}$ & $\begin{array}{l}\text { Estas prácticas buscan dar } \\
\text { oportunidades a quienes ya } \\
\text { conforman los equipos de } \\
\text { gestión, depositando confianza } \\
\text { en ellos, partiendo de la base } \\
\text { que sostenedores solicitan a } \\
\text { los directores mantener los } \\
\text { equipos debido a la rigidez } \\
\text { contractual de los profesionales } \\
\text { docentes y no docentes de la } \\
\text { educación municipal. Posiciona } \\
\text { en los discursos más que } \\
\text { una crítica a la rigidez, una } \\
\text { oportunidad para modificar } \\
\text { responsabilidades desde un } \\
\text { enfoque propio del liderazgo } \\
\text { distribuido (Spillane, 2005). }\end{array}$ & $\begin{array}{l}\text { Ajustar el equipo } \\
\text { de gestión desde } \\
\text { un liderazgo } \\
\text { distribuido } \\
\text { (13 referencias } \\
\text { globales) }\end{array}$ & $\begin{array}{l}\text { "Mira, como director, para que esta cuestión funcione, } \\
\text { unas cosas es el decir y otra cosa es el hacer. Entonces } \\
\text { si yo digo que vamos a hacer una escuela inclusiva, } \\
\text { si vamos a diseñar un plan de gestión inclusivo, yo } \\
\text { no puedo dejar de ser un director inclusivo, no puedo } \\
\text { generar una gestión segregadora, por ejemplo, ¿no } \\
\text { puedo hacer yo grupos con mis favoritos... eehh... te } \\
\text { fijas o no? Y eso pasa, porque acá también es una de } \\
\text { las cosas que encontré en esta escuela, que hay mucho } \\
\text { grupo" (E2). }\end{array}$ \\
\hline
\end{tabular}




\begin{tabular}{|c|c|c|c|c|}
\hline Dimensiones & $\begin{array}{l}\text { Prácticas de } \\
\text { Liderazgo } \\
\text { Inclusivo }\end{array}$ & Justificación ¿Por qué? & $\begin{array}{c}\text { Fines/objetivos } \\
\text { ¿Para qué? }\end{array}$ & Ejemplo extracto de transcripción \\
\hline & $\begin{array}{l}\text { Modificar } \\
\text { aplicación de } \\
\text { normas de } \\
\text { convivencia } \\
\text { desde lo } \\
\text { punitivo hacia } \\
\text { un enfoque } \\
\text { de derechos y } \\
\text { debido proceso } \\
\text { (3 referencias } \\
\text { directas) }\end{array}$ & $\begin{array}{l}\text { Buscar influir mediante nuevas } \\
\text { políticas institucionales en } \\
\text { las interacciones entre los } \\
\text { miembros de la comunidad } \\
\text { educativa en virtud de la } \\
\text { adquisición de un enfoque de } \\
\text { derechos. }\end{array}$ & $\begin{array}{l}\text { Ajustar la } \\
\text { organización } \\
\text { desde un enfoque } \\
\text { de Derechos } \\
\text { (13 referencias } \\
\text { globales) }\end{array}$ & $\begin{array}{l}\text { "primero el gran giro: política es cambiar el espíritu del } \\
\text { manual de convivencia clave, sacar el tema castigo por } \\
\text { un tema de acompañamiento. Yo estoy con el estudiante } \\
\text { porque él me escucha a mí pero hay varios que están } \\
\text { alrededor apoyando; segundo, insistir, insistir, insistir } \\
\text { en los aspectos ambientales del proceso de aprendizaje. } \\
\text { Los espacios educan, la actitud del profe' educa, un } \\
\text { profe malhumorado desencantando dentro de la sala, } \\
\text { los chiquillos lo perciben, lo perciben aunque tú no me } \\
\text { creas" (L 4). }\end{array}$ \\
\hline & $\begin{array}{l}\text { Práctica } \\
\text { inclusiva que } \\
\text { asocia trabajo } \\
\text { colaborativo } \\
\text { y PIE ( } 4 \\
\text { referencias } \\
\text { directas) }\end{array}$ & $\begin{array}{l}\text { Potenciar los espacios de } \\
\text { trabajo colaborativo con } \\
\text { acciones que aumenten } \\
\text { desempeño del trabajo PIE } \\
\text { incorpore en la toma de } \\
\text { decisiones de la escuela. }\end{array}$ & $\begin{array}{l}\text { Re significar la } \\
\text { estrategia de } \\
\text { apoyo ministerial } \\
\text { PIE ( } 8 \text { referencias } \\
\text { globales) }\end{array}$ & $\begin{array}{l}\text { (...) el año } 2 \text { fue agotador, porque me puse a trabajar } \\
\text { en el área técnica. Y el año } 3 \text { es donde trabajamos, } \\
\text { bueno, seguíamos en lo mismo, fuerte con convivencia, } \\
\text { fuerte con lo curricula. El año } 3 \text { fue el tema del PIE. } \\
\text { Que también cuando llegué era la PIE que yo venía } \\
\text { trabajando, yo después me metí al facebook de la } \\
\text { escuela, estaban todas conectadas... no... Entonces el } \\
\text { año } 3 \text { fue el tema para organizar bien las horas de PIE, } \\
\text { incluso hacíamos consejos chicos, los miércoles era el } \\
\text { consejo formal y dentro de las horas de planificación, } \\
\text { por ejemplo, siempre teníamos el lunes para consejo } \\
\text { chico PIE. Empezamos a tomar también decisiones que } \\
\text { tenían que ver con un diseño curricular de la escuela" } \\
\text { (E6). }\end{array}$ \\
\hline \multirow[t]{2}{*}{$\begin{array}{l}\text { Desarrollo } \\
\text { Profesional } \\
\text { Inclusivo (20) }\end{array}$} & $\begin{array}{l}\text { Capacitar a } \\
\text { docentes y no } \\
\text { docentes en } \\
\text { diversificación } \\
\text { de la enseñanza } \\
\text { como un } \\
\text { proceso gradual } \\
\text { de formación } \\
\text { y ejecución del } \\
\text { Decreto Exento } \\
83 \text { del año } 2015 \\
\text { (4 referencias } \\
\text { directas) }\end{array}$ & $\begin{array}{l}\text { Identifican la necesidad de } \\
\text { desarrollar capacidades } \\
\text { profesionales, ya que } \\
\text { cuestionan las competencias } \\
\text { otorgadas por la formación } \\
\text { inicial y formación continua de } \\
\text { los docentes }\end{array}$ & $\begin{array}{l}\text { Incrementar } \\
\text { conocimientos, } \\
\text { actitudes y } \\
\text { recursos al servicio } \\
\text { de un modelo } \\
\text { inclusivo en todos } \\
\text { los funcionarios } \\
\text { docentes y no } \\
\text { docentes que } \\
\text { trabajan el } \\
\text { interior de los } \\
\text { establecimientos } \\
\text { educacionales } \\
\text { (5 referencias } \\
\text { globales) }\end{array}$ & $\begin{array}{l}\text { "Empezamos a diseñar el trabajo que íbamos a hacer } \\
\text { con los niños. Hice a los profes un test, para ver cómo } \\
\text { era su estilo de aprendizaje. Primero identificarnos } \\
\text { quiénes somos nosotros, cómo aprendemos. El tema es } \\
\text { cómo nosotros aprendemos y como nosotros enseñamos. } \\
\text { La idea es... porque si yo soy visual y voy a educar } \\
\text { a mis chiquillos, voy a intencionar en que ellos sean } \\
\text { visuales. Por último, depende del profe la... si yo enseño } \\
\text { visual, los cabros van a ser todos visuales. Entonces ya, } \\
\text { nos vimos, no evaluamos, ya, fue un momento chistoso } \\
\text { porque fuimos viendo en realidad cómo era cada uno" } \\
\text { (E3). }\end{array}$ \\
\hline & $\begin{array}{l}\text { Formar a } \\
\text { profesores } \\
\text { mediante } \\
\text { experiencias } \\
\text { o situaciones } \\
\text { de aprendizaje } \\
\text { (3 referencias } \\
\text { directas) }\end{array}$ & $\begin{array}{l}\text { Producto de los recursos } \\
\text { limitados y la crítica a las } \\
\text { capacitaciones externas, } \\
\text { que frecuentemente no } \\
\text { están arraigadas en las } \\
\text { vivencias cotidianas de } \\
\text { los establecimientos y su } \\
\text { respectivo contexto, se } \\
\text { han adoptado estrategias } \\
\text { de formación utilizando } \\
\text { las competencias de los } \\
\text { profesionales que interactúan } \\
\text { en la comunidad educativa. }\end{array}$ & $\begin{array}{l}\text { Propiciar espacios } \\
\text { de reflexión } \\
\text { sobre el quehacer } \\
\text { profesional docente } \\
\text { como estrategia } \\
\text { de autoformación } \\
\text { continua que } \\
\text { prepare a los } \\
\text { profesionales } \\
\text { para la atención } \\
\text { a la diversidad (8 } \\
\text { referencias). }\end{array}$ & $\begin{array}{l}\text { "Mira lo que pasa en términos de capacitación: yo he } \\
\text { definido más bien vivir situaciones de experiencia que } \\
\text { de capacitaciones de relato. Traer una capacitación que } \\
\text { haga una charla o tres charlas por un día, no creo que, } \\
\text { en estos ambientes de resultados: Las experiencias, } \\
\text { compartir experiencias o vivirlas me ha dado buenos } \\
\text { resultados," (L3) }\end{array}$ \\
\hline
\end{tabular}




\begin{tabular}{|c|c|c|c|c|}
\hline Dimensiones & $\begin{array}{l}\text { Prácticas de } \\
\text { Liderazgo } \\
\text { Inclusivo }\end{array}$ & Justificación ¿Por qué? & $\begin{array}{l}\text { Fines/objetivos } \\
\text { ¿Para qué? }\end{array}$ & Ejemplo extracto de transcripción \\
\hline \multirow[t]{2}{*}{$\begin{array}{l}\text { Atención a los } \\
\text { aprendizajes } \\
\text { diversos (30) }\end{array}$} & $\begin{array}{l}\text { Acompaña- } \\
\text { miento de direc- } \\
\text { tor y docentes } \\
\text { a estudiantes } \\
\text { con dificultades } \\
\text { (3 referencias } \\
\text { directas) }\end{array}$ & $\begin{array}{l}\text { Otorgar oportunidades a los } \\
\text { estudiantes que se encuentran } \\
\text { en situación de discapacidad } \\
\text { y/o apoyo a estudiantes con } \\
\text { dificultades conductuales } \\
\text { que han sido omitidos de } \\
\text { los procesos reglamentarios } \\
\text { asociados a convivencia y/o } \\
\text { apoyo PIE en determinadas } \\
\text { NEE. }\end{array}$ & $\begin{array}{l}\text { Ejecutar } \\
\text { directamente } \\
\text { respaldo a } \\
\text { estudiantes } \\
\text { focalizados } \\
\text { (13 referencias } \\
\text { globales) }\end{array}$ & $\begin{array}{l}\text { "Por ejemplo, ver que los profesores vivan experiencias } \\
\text { a través de tutorías y yo como director tengo seis, siete } \\
\text { niños que acompaño. Un profesor acompaña a otro } \\
\text { niño que presente alguna dificultad. Y dentro de la } \\
\text { resolución.... del manual de convivencia donde habla de } \\
\text { acompañamiento, yo cambié la figura de un castigo por } \\
\text { una situación de acompañamiento para la superación de } \\
\text { la dificultad." (L1) }\end{array}$ \\
\hline & $\begin{array}{l}\text { Enfocar apoyo } \\
\text { a estudiante } \\
\text { en situación de } \\
\text { discapacidad, } \\
\text { desde el acceso } \\
\text { físico y acceso } \\
\text { al currículum } \\
\text { (4 referencias } \\
\text { directas). }\end{array}$ & & $\begin{array}{l}\text { Ejecutar directa- } \\
\text { mente la imple- } \\
\text { mentación de un } \\
\text { currículum diversi- } \\
\text { ficado ( } 10 \text { referen- } \\
\text { cias globales) }\end{array}$ & $\begin{array}{l}\text { "Yo no me imagino un niño con asperger, con algún } \\
\text { síndrome especial del aprendizaje, en una cocina, en un } \\
\text { especial de turismo u hotelería. Bueno, este año vamos } \\
\text { a implementar un curso especial y nos va a permitir es- } \\
\text { tablecer cuáles son las competencias y habilidades desa- } \\
\text { rrollables al mayor nivel posible para que estas personas } \\
\text { puedan tener acceso a su titulación. Vale decir que, si } \\
\text { un niño elige la especialidad de gastronomía, mención } \\
\text { pastelería, va a ser capaz de llevar a cabo un producto } \\
\text { final en el tiempo. Lo importante es establecer que no } \\
\text { puede ser que un niño llegue a cuarto y yo le mienta, } \\
\text { porque es una cuestión de verdad: tú estás titulado en } \\
\text { gastronomía y como cocinero debes saber hacer una } \\
\text { cazuela. Si no logras hacerla porque tu condición no } \\
\text { te permite llegar al producto final, puedes ser un buen } \\
\text { gestor de cocina, administrador de cocina, un prepara- } \\
\text { dor de producto, un reservador" (L4). }\end{array}$ \\
\hline
\end{tabular}

Instalación de una cultura inclusiva en el centro (22)

\author{
Sensibilizar \\ a docentes, \\ alumnos y \\ apoderados \\ para compren- \\ der la inclusión \\ desde el respeto \\ y la diversidad \\ (8 referencias \\ directas)
}

Caracterizan que sus comunidades no se han caracterizado por tener definido un sello asociado al ámbito de la inclusión, sino más bien recién se encuentran en fase de sensibilizar e instalar prácticas reconocidas desde este enfoque.
Promover en la comunidad educativa la adaptación de un sello inclusivo a objeto de actualizar a todos los actores respecto a los nuevos modelos educativos y estilos de ejercicio de la enseñanza y el aprendizaje, buscando una visión compartida de la institución

(14 referencias globales).
"La dirección o el equipo de gestión hace entender a los docentes que el tema de la inclusión es mas allá que tener a un chico en silla de ruedas. Tenemos también que estar dispuestos a atender a chicos que tienen capacidades distintas. Nuestra labor es formar, ayudar a formar y no a sancionar y castigar y trasladar los problemas a los padres y apoderados en las reuniones. En el diálogo más íntimo directamente con los apoderados y cada vez que tengo la oportunidad de conversar con ellos, les refuerzo el tema no con el término de inclusión, si no con el concepto que hay detrás, de aceptarnos, de respetarnos, de ponernos en el lugar del otro porque todos somos diferentes. Este es el trabajo de hormiguita, que creo que es el que más nos ha dado resultado" (E4)

\section{Discusión}

Las prácticas de liderazgo para la inclusión educativa que declaran realizar los directores nóveles están mayoritariamente vinculadas a rediseñar la organización con foco en lo inclusivo, distribuyendo funciones con equipos de trabajo existentes, modificando normativa sobre convivencia escolar y creando condiciones para un trabajo colaborativo. Para estos directores nóveles tiene gran importancia poseer un clima laboral que permita la cohesión de las percepciones de los miembros de la comunidad escolar, respecto de su trabajo y rol social institucional.

Esto es coincidente con lo planteado por Rodríguez (2006) acerca de la necesidad de mantener la estabilidad del clima laboral e influir en los miembros de la organización respecto a su trabajo, como una estrategia que apuesta por la consolidación a lo largo del tiempo de los directores. Gómez-Hurtado (2012) señala que no basta con actitudes intrínsecas, sino que se deben consagrar modelos institucionales que respalden estas actitudes, que permitan, de este modo, consolidar desde lo normativo nuevas conductas en los responsables del establecimiento (López, Julio, Morales, Rojas \& Pérez, 2014). Debatir, consensuar y validar entre la comunidad educativa el enfoque de derechos, se traduce en materializar esta intencionalidad en documentos orientadores como proyectos educativos y reglamentos de convivencia escolar, de evaluación y de promoción de aprendizajes.

Desde la reflexión crítica, recalcan la ausencia de sellos inclusivos en centros escolares diferenciadores de sus prácticas autónomas. La adquisición como consolidación de sellos se contradice con las políticas públicas genéricas y no necesariamente con las autorías de cada comunidad educativa. Fernández-Batanero (2013) señala, desde un análisis constante de las prácticas educativas y de los procesos de cambio escolar, que no puede reducirse solo a una ley con tiempo limitado, pues se han declarado muchas intenciones y reglamentos que se han implementado en diversos contextos, pero sin la socialización, discusión y reflexión de las implicancias que tiene y que deben asumir los distintos actores educativos en materia de inclusión escolar. 


\section{Conclusiones}

Respecto de cómo los directores noveles dan respuesta a las necesidades derivadas de la inclusión educativa en sus centros escolares, podemos señalar que estos están en una etapa inicial de sensibilización e instalación de prácticas en las escuelas y liceos donde ejercen su liderazgo, donde cobra importancia la organización de equipos de trabajo, la transformación de la normativa de convivencia escolar desde un enfoque punitivo hacia el de derechos, la definición institucional de Proyectos Educativos (PEI) que enfatizan la multiculturalidad y el trabajo colaborativo, es decir prácticas asociadas a rediseñar la organización, pero con ausencia de sellos inclusivos diferenciadores de sus prácticas autónomas, observándose la necesidad de una formación de auténticas comunidades escolares en materia de inclusión educativa, considerando no solo al personal docente del centro, sino también al personal no docente.

A modo de síntesis, se puede decir que:

- Inicialmente, hay un cierto respeto a la cultura institucional establecida, supeditando su transformación al desarrollo de la convivencia, los derechos de los actores y a la gestión del proyecto educativo.

- Con relación a las necesidades educativas las actuaciones se centran más en la adaptación de infraestructuras o gestión del espacio, la diversificación curricular y el fomento de la participación de los estudiantes.

- Al no haber una cultura inclusiva preestablecida, los esfuerzos se orientan principalmente a procesos de sensibilización y ajustes en el diseño y desarrollo del PEI.

- Se destacan otros aspectos relacionados con necesidades de apoyo para la formación permanente del profesorado, la gestión de sus fortalezas y la dimensión socio-emocional.

$\mathrm{Al}$ respecto, podemos concluir que la mirada que tienen los directores nóveles, nos aproxima más a la integración que a la inclusión, obviando el valor de la diversidad como elemento enriquecedor del proceso de enseñanza y aprendizaje y como factor que favorece el desarrollo humano desde la multidimensionalidad y no solo desde la discapacidad.

Finalmente, la principal limitante de esta investigación es que sólo se examinan prácticas inclusivas de directores nóveles desde lo que declaran qué hacen y no desde lo que hacen realmente. De todas formas, los aportes de este estudio se orientan a proporcionar a las políticas educativas de una serie de conjeturas emergentes dirigidas a apoyar una base formativa previa para los directores y con un enfoque inclusivo. Supondría, dotarles de una base técnica y teoría concordante con las políticas de resguardo de derechos que se buscan instalar y consolidar en las comunidades educativas.

\section{Agradecimientos:}

Investigación Financiada por Conicyt, Proyecto Fondecyt XXXX del Gobierno de Chile.

\section{Referencias Bibliográficas}

Ainscow, M. (2006). Towards a More Inclusive Education System: where next for special schools? En Cigman, R. (Edit.). Included or Excluded? The challenge of the Mainstream for some SEN Children. (p. Forthcoming). London: Routledge.

Alonso, L. (1998) La mirada cualitativa en sociología: una aproximación interpretativa. Madrid: Editorial Fundamentos.
Apablaza, M. (2014). Representaciones Sociales de profesores respecto de la diversidad escolar en relación a los contextos de desempeño profesional, prácticas y formación inicial. Revista Estudios Pedagógicos, 40(1), 7-14. doi 10.4067/S071807052014000100001

Arnáiz, P. (2003). Educación inclusiva: Una educación para todos. España: ALJIBE

Arnáiz, P. (2010, septiembre). Educación de Calidad en una escuela para todos. Conferencia presentada en el Congreso Internacional Tecnoneet, Murcia, España.

Blanco, G. (2006). La Equidad y la inclusión social: Uno de los desafíos de la educación y la escuela hoy. Revista Iberoamericana sobre Calidad, Eficacia y Cambio en Educación, 4(3), 1-15. Recuperado de http://www.redalyc.org/pdf/551/Resumenes/ Resumen_55140302_1.pdf

Bolívar, A., López, J. \& Murillo, F.J. (2013). Liderazgo en las instituciones educativas: Una revisión de líneas de investigación. Revista Fuentes, 14, 15-60. Recuperado de http://institucional. us.es/revistas/fuente/14/Firma\%20invitada.pdf

Bourdieu, P. (2005). Capital cultural, escuela y espacio social. Buenos Aires: Siglo XXI

Booth, T. \& Ainscow, M. (2012) Guía para la inclusión educativa: Desarrollando el aprendizaje y la participación en las escuelas. Santiago de Chile: CSIE-FCF

Carrasco, A. \& González, P. (2017). Liderazgo para la inclusión y para la justicia social. Educación y Ciudad, 33, 63-74. Recuperado de http://www.idep.edu.co/revistas/index.php/educacion-y-ciudad/article/view/1648

Day, C., Sammons, P., Leithwood, K., Hoptkins, D., Harris, A., Gu, Q. y Brown, E. (2010). Ten Strong Claims About Successful School Leadership. Nottingham: NCSL.

Echeita, G. (2007). Evaluación sistemática de un proyecto de innovación para atender a la diversidad del alumnado en la educación secundaria obligatoria: Aula cooperativa multinivel. Revista Iberoamericana sobre Calidad, Eficacia y Cambio en Educación, 5(3), 152-172. Recuperado de http://www.redalyc. org/pdf/551/55130512.pdf

Echeita, G. (2010). La Educación inclusiva como derecho. Marco de referencia y pautas de acción para el desarrollo de una revolución pendiente. Recuperado de https://www.uam.es/personal_pdi/ stmaria/sarrio/DOCUMENTOS, \%20ARTICULOS, \%20PONENECIAS,/Educacion $\% 20$ inclusiva $\% 20$ como $\% 20$ derecho. $\% 20$ Ainscow $\% 20 y \% 20$ Echeita.pdf

Echeita, G. \& Ainscow, M. (2011). La educación inclusiva como derecho: marco de referencia y pautas de acción para el desarrollo de una revolución pendiente. Tejuelo. Didáctica de la Lengua y la Literatura, 12, 26-46. Recuperado de http://dehesa.unex.es/bitstream/handle/10662/4644/1988-8430_12_26.pdf?sequence=1

Fernández, J. \& Hernández, A. (2013). Liderazgo directivo e inclusión educativa. Estudio de Casos. Perfiles Educativos, 35(142), 27-41. doi: 10.1016/S0185-2698(13)71847-6

Fernández-Batanero, J. (2013). Competencias docentes y educación inclusiva.Redie. Revista Electrónica de Investigación Educativa. 15(2), 82-99. Recuperado de https://redie.uabc.mx/redie/ article/view/445/610

Figueroa, I. \& Muñoz, Y. (2014). La guía para la inclusión educativa como herramienta de autoevaluación institucional: Reporte de una experiencia. Revista Latinoamericana de Educación Inclusiva, 8(2), 179-198. Recuperado de https://www.researchgate.net/publication/305703121_La_Guia_para_la_Inclusion_Educativa_como_herramienta_de_autoevaluacion_institucional_Reporte_de_una_experiencia

Gairín, J. (2010). Gestionar la complejidad en los centros educativos actuales. En M. Gómez (Coord.). El liderazgo educativo. 
Los equipos directivos en centros de secundaria, elementos básicos del éxito escolar (79-92). Madrid: Ministerio de Educación, Servicio de Publicaciones.

Gairín, J. (2015) (Coord.). Acceso, permanencia y éxito académico de colectivos vulnerables en la Educación Superior. Estrategias para la intervención. Barcelona: UAB. Edo Serveis.

Gómez-Hurtado, I. (2012). Una Dirección escolar para la inclusión escolar. Revista Perspectiva Educacional, formación de profesores, 51(2), 21-45. doi: 10.4151/07189729-Vol.51-Iss.2-Art.108

Gómez-Hurtado, I. (2014). Dirección y Gestión de la Diversidad en la Escuela. Hacia un liderazgo Inclusivo. Revista Fuentes, 14, 61-84. Recuperado de http://institucional.us.es/revistas/ fuente/14/Direcci\%C3\%B3n\%20y\%20gesti\%C3\%B3n.pdf

González, M. (2008). Diversidad e inclusión educativa: Algunas reflexiones sobre el liderazgo en el centro escolar. REICE Revista Electrónica Iberoamericana sobre Calidad Eficacia y Cambio en Educación, 6(2) 2, 82-99. Recuperado de https://www. redalyc.org/pdf/551/55160208.pdf

Gronn, P. (2002). Distributed Leadership as a Unit of Analysis. The Leadership Quarterly, 13(4), 423-451. doi: 10.1016/S10489843(02)00120-0

Guerrero, M., \& Urbano, D. (2007): Entrepreneurial Universities: The Case of Autonomous University of Barcelona, Barcelona: Universitat Autonoma de Barcelona.

Hallinger, P. \& Wang, W. C. (2015). Assessing Instructional Leadership with the Principal Instructional Management Rating Scale. Dordrecht, Netherlands: Springer.

Informe Warnock. (1978) Sobre educación especial: Special Education's Needs [Documento]. Recuperado de https://pdfs. semanticscholar.org/1d03/62c1713e075c2dba48f95f83e6195bc8e7d9.pdf

Laluvein, J. (2010). Parents, Teachers and the "Community of Practice". The Qualitative Report, 15(1), 176-195. Recuperado de https://nsuworks.nova.edu/tqr/vol15/iss1/10/

Leithwood, K. (2009) ¿Cómo liderar nuestras escuelas? Aportes desde la investigación. Santiago de Chile: Fundación Chile.

Leithwood, K. \& Louis, K.S. (2011). Linking Leadership to Student Learning. San Francisco: Jossey-Bass.

Leithwood, K. \& Riehl, C. (2005). What do we Already Know About Educational Leadership? En W. Firestone, \& C. Riehl (Eds.). A New Agenda for Research in Educational Leadership (pp. 12-27). Nueva York: Teacher College Press.

Leiva, M.V., Montecinos, C., Ahumada, L., Campos, F. \& Guerra, S. (2017). Novice Principals' Instructional Management Practices in High Poverty, Low Performing Schools in Chile. Revista Procedia, 237, 3-9. doi: 10.1016/j.sbspro.2017.02.003

López, V., Julio, C., Morales, M., Rojas, C. \& Pérez, M. (2014). Barreras culturales para la inclusión: políticas y prácticas de integración en Chile. Revista de Educación, 363, 256-281. doi: 10.4438/1988-592X-RE-2012-363-180

Marcelo, C., Murillo, P., Sánchez, M., Mayor, C. \& Rodríguez, J. (septiembre, 2010). Compilación de diseños de aprendizaje en el repositorio Alacena. En B. Águeda (Presidente). VII Jornadas Internacionales de Innovación Universitaria. Universidad Europea de Madrid, Madrid, España.

Martínez-Figueira, E. (2013) Una mirada hacia la inclusión: barreras en el camino a la participación. Revista Perspectiva Educacional, Formación de Profesores, 52(2), 177-200. doi: 10.4151/07189729-Vol.52-Iss.2-Art.101

Mayring, P. (2000). Qualitative Content Analysis. Forum Qualitative Sozialforschung, 1(2). doi: 10.17169/fqs-1.2.1089

Merrian, S. (2002). Qualitative Research in Practice. San Francisco: Jossey-Bass.
Murillo J., Krichesky, G., Castro A. \& Hernández R. (2010). Liderazgo para la inclusión escolar y la justicia social. Aportaciones de la investigación. Revista Latinoamericana de Inclusión Educativa, 4(1), 169-186.

Muijs, D., Harris, A., Chapman, C., Stoll, L. \& Russ, J. (2004). Improving Schools in Socio-Economically Disadvantaged Areas: A Review of Research Evidence. School Effectiveness and School Improvement, 15(2), 149-175 doi: 10.1076\%2Fsesi.15.2.149.30433

Murillo, F.J. \& Hernández, R. (2011). Una dirección escolar para la inclusión. Revista de Organización y Gestión Educativa, 19(1), 17-21.

Organización de las Naciones Unidas para la Educación, la Ciencia y la Cultura. (2011). Educación Inclusiva. Santiago, Chile: Organización de las Naciones Unidades para la Educación, la Ciencia y la Cultura. Recuperado de http://www.unesco.org/ new/es/santiago/education/inclusive-education/

Ossa, C. (2014). Integración Escolar: ¿Cambio para el alumno o cambio para la escuela? Revista de Estudios y Experiencias en Educación, 13(25), 153- 164. Recuperado de http://www.rexe. $\mathrm{cl} / 25 /$ pdf/art.9.pdf

Parrilla, A. (2007). Inclusive Education in Spain: A View from Inside. En: L. Barton and F. Amstrong (Eds.) Policy, Experience and Change: Cross Cultural Reflections on Inclusive Education (pp.19-36). Dordrecht: Springer.

Reyes, R. (2002). Diccionario Crítico de Ciencias Sociales. Madrid: Ed. Universidad Complutense.

Robinson, V., Hohepa, M. \& Lloyd, C. (2009). School Leadership and Student Outcomes: Identifying what Works and Why. Best Evidence Syntheses Iteration (BES). New Zealand: Ministry of Education.

Robinson, V., Lloyd, C. \& Rowe, K. (2008). The Impact of Leadership on Student Outcomes: An Analysis of the Differential Effects of Leadership Types. Educational Administration Quaerterly, 44(5), 635-674. doi: 10.1177/0013161X08321509

Rodríguez, D. (2006). Modelos para la creación y gestión del conocimiento: una aproximación teórica. Revista Educar, 37, 25-39. Recuperado de https://ddd.uab.cat/pub/educar/0211819Xn37/0211819Xn37p25.pdf

Rodríguez, F. \& Ossa, C. (2014) Valoración del trabajo colaborativo entre profesores de escuelas básicas de Tomé, Chile. Estudios Pedagógicos, 40(2), 303-319. doi: 10.4067/S071807052014000300018

Simón, C. \& Barrios, A. (2019). Las familias en el corazón de la educación inclusiva. Aula Abierta, 48(1), pp. 51-58. DOI: https://doi.org/10.17811/rifie.48.1.2019.51-58

Spillane, J.P. (2005). Distributed Leadership. The Educational Forum, 69(2), 143-150. Doi: 10.1080/00131720508984678

Spillane, J.P.,Halverson. R., \& Diamond J.B.(2004). Towards a theory of leadership Practice: A distributive perspective. Journal of Curriculum Studies, 36(1), 3.34 Doi: 10.1080/0022027032000106726

UNESCO (2005) Guidelines for inclusión: Ensuring Access to Education for All. París: UNESCO (Accesible on line en: http://unesco.org/educacion/inclusive)

Valles, M. (1999). Técnicas cualitativas de investigación social. Reflexión metodológica y práctica profesional. Madrid: Síntesis.

Weinstein, J. (2009). Liderazgo directivo, asignatura pendiente de la reforma educacional chilena. Revista Estudios Sociales 117, 123-148. Recuperado de http://www.educandojuntos. cl/wp-content/uploads/2015/11/liderazgo-directivoasignatura-pendiente-de-la-reforma-educacional-chilena-jose-weinstein.pdf 
